\title{
Observing other universe through ringholes and Klein-bottle holes
}

\author{
Pedro F. González-Díaz and Ana Alonso-Serrano \\ Colina de los Chopos, Instituto de Física Fundamental, \\ Consejo Superior de Investigaciones Científicas, Serrano 121, 28006 Madrid, Spain
}

\begin{abstract}
It is argued that whereas the Shatskiy single rings produced by the gravitational inner field of a spherically symmetric wormhole and the concentric double Einstein rings generated by a toroidal ringhole could not be used without some uncertainty to identify the presence of such tunnelings in the universe or the existence of a parallel universe, the image which the inner gravitational field of a non orientable Klein-bottle hole tunneling would leave by lensing a single luminous source is that of a truncated double spiral, which is a signature that cannot be attributed to any other single or composite astronomical object in whichever universe it may be placed. In this report we argue some more reasons to predict that such a signature would imply the discovery of one such non orientable tunneling in our or other universe. After all, a nonorientable Klein-bottle hole is also a perfectly valid solution to the Einstein equations and the stuff which would make it feasible is becoming more and more familiar in cosmology.
\end{abstract}

PACS numbers: 95,30.sf, 04.40.-b

\section{INTRODUCTION}

Considering observable effects from wormholes or ringholes is not new. More than a decade ago some of such effects were already predicted by several author $[1,2]$. In particular, it was shown that these tunnelings can induce lensing effects from luminous sources. More precisely, by embedding these tunnelings in Friedmann space, it was seen [2] that, besides the expected lensing, at least ringholes were able to induce other potentially observable effects such as frequency shifting of the emitting sources, discontinuous change of background temperature, broading and intensity enhancement of the spectral lines, as well as a rather dramatic increase in the luminosity of any objects at the tunnel throat. Moreover, the precise form of the lensing signature left by wormholes and ringholes has been quite more recently seen to consist of a single ring and a double concentric ring, respectively $[3,4]$. In spite of the interest that all of such results may have from a pedagogical standpoint, none of these induced phenomena has by itself any practical usefulness in order to identify the existence of space-time tunnels in the universe because there are other observable objects in the universe able to produce similar effects.

In particular, some hope was raised in using wormholes and specially ringholes to get a direct evidence of the existence of other universes to which they could be connected, so traversing some information from them to ours own. Nevertheless, such a hope is easily shown to vanish when the following two arguments are taken into account. First of all, the above alluded uncertainty that the effects be produced by these tunnelings and not by more familiar astronomical objects such as galaxies, stars black holes or quasars.

On the other hand, even though some authors have tried to consider models where other universes were made observable to us through collision with our own [5]. Moreover, from the very definition of universe it follows that provided that there cannot be any space-time connec- tions between whichever pair of universes, less yet it is allowed the possibility for any well-defined relation between the spaces or times of them. It could be argued that if one allows the connecting wormholes or ringholes to be converted into time machines with completely unspecified mutual velocities between the mouths one might create a whole space-time that would represent two universes. However, such a possibility cannot be entirely implemented because such a whole space-time would be orientable against the opposite evidence that it would necessarily violate orientability. Thus, rather than using orientable space-time tunnels such as wormhole or ringholes, it appears that two universes can be mutually tunneled to each other while preserving their property of still being well-defined universes if one by instance used non orientable Klein bottle holes converted into time machines by allowing their mouths to move relative to one another at completely unspecified speeds [6]. In this paper we shall consider the effects that such connections would produce and the possibility to using them to check the existence of universes other than ours.

One of the most important revolutions in cosmology is taking place right now, that of the so called multiverse [7]. In order to convert this revolution in more than just a speculative idea, providing it with a physical content, one should try to consider alternate ways which would for example include the effects that time machines derived from nonorientable Klein bottle holes [6] may have on the luminous sources placed beyond the limit of our universe. Something that any of the existing multiverse theories is very needed of. In fact, Linde ideas coming about string theories and alike [8] or other's ideas [9] may make the notion of a multiverse more plausible, but they do not prove that other universes are really out there. The staggering challenge is to think of a conceivable experiment or observation confined to our own universe based on looking for some footprints left by nonorientable tunnelings connecting our universe to other universes. General principles of physics cast in fact serious doubts on whether it make 
sense to talk about other universes if they can never be detected.

Rees, an early supporter of multiverse idea, agrees [10] that it may never be possible to observe other universes directly, but he argues that scientists may still be able to make a convincing case for their existence. To do that, he says, physicists will need a theory of the multiverse that makes new but testable predictions about properties of our own universe. If, similarly to as current observations have confirmed big bang as a well established model, new experiments coming perhaps from Large Hadron Collider or the Planck satellite space mission, indirectly confirmed such a theory predictions about the universe we can see, Rees believes, they would also make a strong case for the reality of those we cannot. String theory is still very much a work in progress, but it could form the basis for the sort of theory that Rees is calling for.

However, the very essentials of quantum theory show some great, almost insurmountable odds against the Rees philosophy, specially if one adheres to the quantumcosmological ideas that support the principle according to which physical reality should be directly observable or it vanishes into nothing. In this way, it appears a casus of full necessity to explore the existing ways that may lead us to directly observe a property or characteristic of a universe other than ours, such as the main objective of the present paper is aiming at exploring the possible existence of other universes through a search for the lensing signature of orientable ringholes and non orientable Klein bottle holes connecting us to other universe.

\section{WORMHOLE SIGNATURE}

It was first noted by Shatskiy [3] that wormholes, which are usually disguised as black holes, can be made observable and recognizable in terms of bright, glowing rings originating from the necessary flaring out of the embedding surface around their throat which is produced by the presence of the so called phantom stuff [11]. The really most devastating argument against the proposed wormhole distinguishable character of the Shatskiy rings is that, even if exotic matter does exist, other many objects are able to create a similar lensing light signature [12]. In particular, while the orientability of the wormhole manifold makes it impossible using these solutions for observing any thing from a universe other than ours, it is hard to see how the resulting lensing rings could be differentiated from the astronomical blueprint left by negative energy stars and, mainly, from all those massive astronomical objects, such as galaxies, black holes or quasars, whose gravitational lensing effects appear as the so called Einstein rings [11].

The actual problems are with the symmetry and the orientability of the throat. Wormholes are orientable manifolds which are characterized by a spherically symmetric throat and, therefore, there will be a diverging lensing effect undergone by any bundle of light rays com-

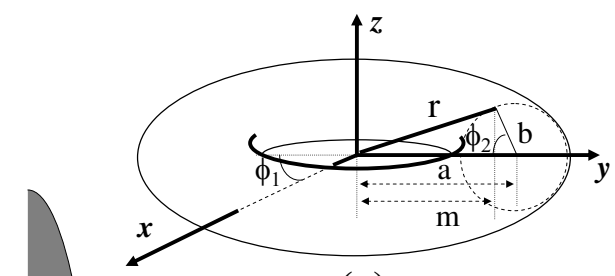

(a)

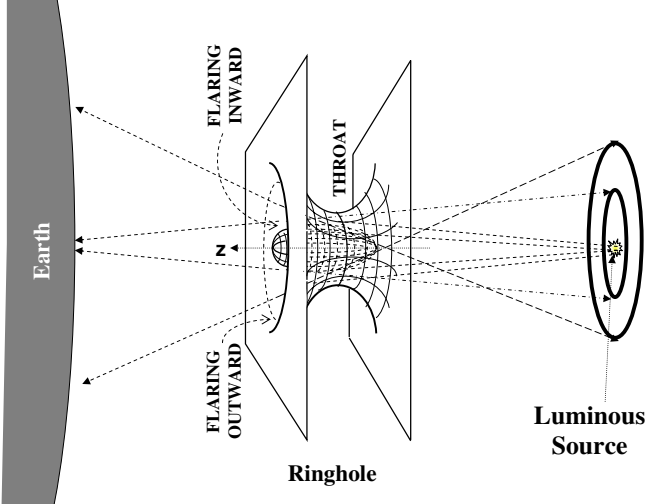

(b)

FIG. 1: Gravitational lensing effect produced by a ringhole from a single luminous source. (a) Parameters defining the toroidal ringhole throat in terms of which metric (1) is defined. (b) Rays passing near the outer and inner surfaces respectively flare outward and inward, leading to a image from a luminous point placed behind the ringhole which is made of two concentric bright rings. The relative mutual positions of these rings would depend on the distance between the ringhole and the luminous source. If that distance is small enough then the larger outer ring comes from the flaring inward surface, and conversely, if the distance source-ringhole is increased then the outer ring comes from the outward surface, the larger that distance the greater the difference between the two bright rings.

ing from a luminous source placed behind the furthest wormhole mouth which would necessarily manifest to observers on Earth as single perfectly circular rings, such as it was indicated by Shatskiy [3]. This pattern could well be misinterpreted as being originated from a star or other massive astronomical object necessarily placed in our own universe, instead of a wormhole, with a radius quite smaller than that for that wormhole throat radius.

\section{RINGHOLE SIGNATURE}

An inner tunneling symmetry which would give rise to a more distinguishable lensing pattern is that of a ringhole [13], that is, an orientable space-time tunnel whose 
throat has the toroidal symmetry, instead of the spherical symmetry. Using the set of geometrical parameters specified in the upper part of Fig. (1) we can derive the metric for a ringhole to be [13]

$$
d s^{2}=-C_{2} r^{2} d t^{2}+b^{2}\left[1+\frac{C_{1} a^{2} \sin ^{2} \varphi_{2}}{r^{6}\left(1-\frac{A^{2}}{r^{4}}\right)}\right] d \varphi_{2}^{2}+m^{2} d \varphi_{1}^{2}
$$

where

$A=a^{2}-b^{2}, \quad m=a-b \cos \varphi_{2}, \quad r=\sqrt{a^{2}+b^{2}-2 a b \cos \varphi_{2}}$,

with $C_{1}$ and $C_{2}$ arbitrary integration constants, and $a$ and $b$ the radius of the circumference generated by the circular axis of the torus and that of a torus section, respectively, with $a>b$. Metric (1) is defined for $0 \leq$ $t \leq \infty, a-b \leq r \leq a+b$ and the angles (see Fig. 1 (a)) $0 \leq \varphi_{1}, \varphi_{2} \leq 2 \pi$.

A thorough analysis $[4,13]$ leads then us to deduce that one would expect lensing effects to occur at or near the ringhole throat, that is to say, the mouths would act like a diverging lens for world lines along $2 \pi-\varphi_{2}^{c}>\varphi_{2}>\varphi_{2}^{c}$, and like a converging lens for world lines along $-\varphi_{2}^{c}<$ $\varphi_{2}<\varphi_{2}^{c}$. No lensing actions would therefore take place at $\varphi_{2}=\varphi_{2}^{c}$ and $\varphi_{2}=2 \pi-\varphi_{2}^{c}$. In fact, in the case of ringholes, instead of producing just a single flaring outward for light rays passing through the wormhole throat, this multiply connected topology, in addition to that flaring outward (diverging) effect, also produces a flaring inward (converging) effect ${ }^{13}$ on the light rays that pass through its throat, in such a way that an observer on Earth would interpret light passing through the ringhole throat from a single luminous source as coming from two bright, glowing concentric rings, which form up the distinctive peculiar pattern from ringholes (See Fig. 1 (b)). That pattern cannot be generated by any other possible disturbing astronomical object other than a very implausible set of three luminous massive objects (let us say galaxies) which must be so perfectly aligned along the sigh line that its occurrence becomes extremely unlikely.

It is readily inferred from Fig. 1 (b) that, for a reasonably large ringhole sufficiently far from the luminous source, the inner bright ring would correspond to the flaring inward (converging) surfaces. If we keep the ringhole size invariant and the distance between the ringhole and the luminous source is decreased drastically, then the inner bright ring would turn to be produced by the flaring outward (diverging) surface.

Such a ringhole signature may have been already observed though it has been so far attributed to the combined effect of two Einstein rings originated from the above-considered to be extremely unlikely superprecise alignment of three galaxies. In fact, at the beginning of 2008 The NASA/ESA Hubble Space Telescope revealed [14] a never-before-seen phenomenon in space: a pair of glowing rings, one nestled inside the other like a bull'seye pattern. This double-ring pattern was interpreted as a double Einstein ring being caused by the complex bending of light from two distant galaxies strung directly behind a foreground massive galaxy, like three beads on a string along the line of sight, simply because at the time there were no other available interpretations for what was being observed. Being more than just a novelty, this very rare phenomenon found with the Hubble Space Telescope could, moreover, eventually offer insight into dark matter, dark energy, the nature of distant galaxies, and even the curvature of the Universe.

As previously stated, for that interpretation to be feasible, the massive foreground galaxy had to be almost perfectly aligned in the sky with two background galaxies at different distances to justify the finding. The foreground galaxy is 3 billion light-years away. Now, in order to justify the ratio between the two ring radii, the inner ring and outer ring would be comprised of multiple images of two galaxies at a distance of some 6 billion and approximately 11 billion light-years.

However, the odds of observing the required extremely precise alignment of the three galaxies are so small (an estimated 1 in 10,000) that even some of the discoverers of that astronomical phenomena said that they had 'hit the jackpot' with the discovery. At the time, the authors of Ref. 14 had no alternative other than accepting that quite improbable interpretation of the result. Nevertheless, having we uncovered that such concentric rings may well be also interpreted as the blueprint of the presence of a ringhole in the direction in space where the double bright ring system was discovered, we may also adopt the latter interpretation in terms of a ringhole as an alternate possible explanation for that phenomenon, taking now the luminous sources at redshifts corresponding to 3 and 6 billion light-years as measuring the positions of the two ringhole mouths on the sky (provided the mouths are surrounded by some sufficiently large quantities of luminous material), and their respective luminosities as stemming from the respective light deflections along the angle $\varphi_{2}$ caused by the combined effect of the size of the throat radius and the relative distance between the two mouths. Even in the absence of sufficiently high matter densities around the two ringhole mouths in a still large enough tunneling, since the two rings have the same spectra in the case of a ringhole, it could well be that the unseen second source might be too faint so that these spectra actually be the same which corresponds to matter placed anywhere along the ringhole tunneling. The feature that the surface brightness of the two rings would be different may also be justified by the above-suggested different distribution of ordinary and exotic matter leading to distinct absorption and dispersion effects of the incoming light along the two horizon separated $\varphi_{2}$-angular regions around the throat. In any event, because of the orientability of the single ringhole manifold or the composite manifold formed by the aligned galaxies, which is required to get the observed concentric double ring bright image, the luminous source originating the lensing effect can never be placed in a universe other than ours, making in this way a ringhole a completely useless tool to check 
the possible existence of other universes.

\section{KLEIN BOTTLE SIGNATURE}

The use of inter-universe tunnelings in order to check the existence of other universes should actually require choosing spacetime holes which be (i) non orientable and (ii) convertible into a time machine with completely unspecified relative speed between its mouths. One such space-time construct has in fact been already studied, in the shape of what is dubbed a Klein bottle hole [6]. In this case, non orientability is guaranteed by the existence of a throat with the topology of a Klein bottle and it was also shown that this space-time can be stable to vacuum fluctuations and is also convertible into a time machine with fully arbitrary inter mouths speed. Employing the set of geometrical parameters specified in Fig. 2 and in the lower part of Fig. 3 we can derive the space-time metric for one of such Klein bottle holes to be [6]

$$
\begin{aligned}
& d s^{2}=-e^{2 \Phi} d t^{2}+\theta\left(2 \pi-\varphi_{1}\right)\left(\frac{d r_{1}^{2}}{1-K\left(b_{1}\right) / b_{1}}+d \Omega_{1}^{2}\right) \\
& +\theta\left(\varphi_{1}-2 \pi\right)\left(\frac{d r_{2}^{2}}{1-K\left(b_{2}\right) / b_{2}}+d \Omega_{2}^{2}\right),
\end{aligned}
$$

where the $\theta(x)$ 's are the step Heaviside function [15], with $\theta(x)=1$ for $x>0$ and $\theta(x)=0$ for $x<0, d \Omega_{i}^{2}$ 's for $0 \leq \varphi_{1} \leq 2 \pi$ is given by

$$
\begin{aligned}
& d \Omega_{1}^{2}=\left\{m_{1}^{2}+\frac{1}{4}\left[M_{1}\left(a_{1}-C_{1}\right)+N_{1}\left(b_{1}-D_{1}\right)\right]\right\} d \varphi_{1}^{2} \\
& b_{1}^{2} d \varphi_{2}^{2}-b_{1} \sqrt{\left(a_{1}-C_{1}\right)\left(A_{1}-a_{1}\right)} \sin \varphi_{2} d \varphi_{1} d \varphi_{2}
\end{aligned}
$$

in which

$$
\begin{gathered}
M_{1}=A_{1}-a_{1}-\left(B_{1}-b_{1}\right) \cos \varphi_{2} \\
N_{1}=B_{1}-b_{1}-\left(A_{1}-a_{1}\right) \cos \varphi_{2} \\
m_{i}=a_{i}-b_{i} \cos \varphi_{2}, \quad i=1,2
\end{gathered}
$$

Now, for $2 \pi \leq \varphi_{1} \leq 3 \pi$

$$
\begin{aligned}
& d \Omega_{1}^{2}=\left\{m_{2}^{2}+\frac{1}{4}\left[M_{2}\left(a_{2}-A_{2}\right)+N_{2}\left(b_{2}-B_{2}\right)\right]\right\} d \varphi_{2}^{2} \\
& b_{2}^{2} d \varphi_{2}^{2}-b_{2} \sqrt{\left(a_{2}-A_{2}\right)\left(C_{2}-a_{2}\right)} \sin \varphi_{2} d \varphi_{1} d \varphi_{2}
\end{aligned}
$$

where in this case

$$
\begin{aligned}
& M_{2}=C_{2}-a_{2}-\left(D_{2}-b_{2}\right) \cos \varphi_{2} \\
& N_{2}=D_{2}-b_{2}-\left(C_{2}-a_{2}\right) \cos \varphi_{2} .
\end{aligned}
$$

Finally we have

$$
r_{1}=\sqrt{a_{1}^{2}+b_{1}^{2}-2 a_{1} b_{1} \cos \varphi_{2}}
$$

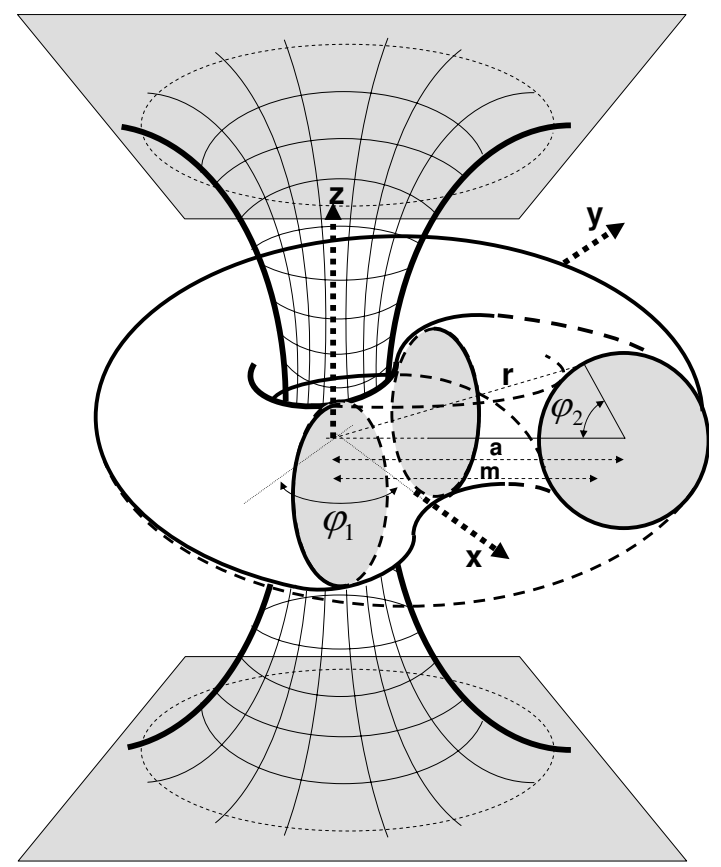

FIG. 2: Pictorial representation of a Klein bottle hole showing the non orientable topology of its throat and some of the parameters in terms of which metric is defined (see also Fig. 3).

$$
r_{2}=\sqrt{a_{2}^{2}+b_{2}^{2}+2 a_{2} b_{2} \cos \varphi_{2}}
$$

where we have extended the range of the angular coordinate $\varphi_{1}$ to also encompass values continuously running from $2 \pi$ to $3 \pi$ and $A_{i}, B_{i}, C_{i}$ and $D_{i}, i=1,2$, are given sets of adjustable parameters which are arbitrary unless for the conditions $A_{1}>C_{1}, B_{1}>D_{1}, A_{1}>B_{1}$ and $C_{1}>C_{1}$ for the angular interval $0 \leq \varphi_{1} \leq 2 \pi$, whereas for $2 \pi \leq \varphi_{1} \leq 3 \pi$ we must have $C_{2}>A_{2}, D_{2}>B_{2}$, $C_{2}>D_{2}$ and $A_{2}>B_{2}$, with $D_{2}=B_{!}, B_{2}=D_{1}$ and $A_{1}-C_{1}=A_{2}+C_{2}$, with $A_{1}-C_{1}>2 A_{2}$. $a_{i}$ and $b_{i}$ are the radius of the circumference generated by the circular axis of the Klein bottle torus and that of a Klein bottle section, respectively, with $a_{i}>b_{i}$. Metric (3) is defined for $0 \leq t \leq \infty, a_{i}-b_{i} \leq r_{i} \leq a_{i}+b_{i}$ and the angles (see Figs. 2 and 3) $0 \leq \varphi_{1}, \varphi_{2} \leq 2 \pi$.

In order to check the properties of a Klein bottle hole as a lens, we now write the static space-time metric of a single, traversible Klein bottle hole in the form

$$
d s^{2}=-d t^{2}+\theta\left(2 \pi-\varphi_{1}\right)\left[\left(\frac{n_{1}\left(\ell_{1}\right)}{r_{1}\left(\ell_{1}\right)}\right)^{2} d \ell_{1}^{2}+d \Omega_{1}^{2}\left(\ell_{1}\right)\right]
$$




$$
+\theta\left(\varphi_{1}-2 \pi\right)\left[\left(\frac{n_{2}\left(\ell_{2}\right)}{r_{2}\left(\ell_{2}\right)}\right)^{2} d \ell_{2}^{2}+d \Omega_{2}^{2}\left(\ell_{2}\right)\right]
$$

where $-\infty<t<+\infty$, with $-\infty<\ell_{i}<+\infty$, and $i=$ 1,2 . Here, $b_{i}$ is replaced for $\sqrt{\ell_{i}^{2}+b_{0}^{2}}, \ell_{i}$ being the proper radial distance of each transversal section of the Klein bottle on the respective $\varphi_{1}$ interval for $i$, and

$$
\begin{gathered}
m_{i}\left(\ell_{i}\right)=a_{i}-\left(\ell_{i}^{2}+b_{0 i}^{2}\right)^{1 / 2} \cos \varphi_{2} \\
n_{i}\left(\ell_{i}\right)=\left(\ell_{i}^{2}+b_{0 i}^{2}\right)^{1 / 2}-a_{i} \cos \varphi_{2}, \\
r_{i}\left(\ell_{i}\right)=\sqrt{a_{i}^{2}+\ell_{i}^{2}+b_{0 i}^{2}+2(-1)^{i}\left(\ell_{i}^{2}+b_{0 i}^{2}\right)^{1 / 2} a_{i} \cos \varphi_{2}},
\end{gathered}
$$

in which $b_{0 i}$ is as given from $b_{1}=\left(B_{1}-D_{1}\right) \cos ^{2}\left(\varphi_{1} / 4\right)+$ $D_{1}$ and $b_{2}=\left(D_{2}-B_{2}\right) \sin ^{2}\left(\varphi_{1} / 2\right)+B_{2}$ for constant parameter adjusted to be the radius of the throat of the Klein bottle hole at $\ell_{i}=0$. As $\ell_{i}$ increases from $-\infty$ to $0, b_{i}$ decreases monotonously from $+\infty$ to its minimum value $b_{0 i}$ at the throat radius, and as $\ell_{i}$ increases onward to $+\infty, b_{i}$ increases monotonously to $+\infty$ again. Now, for metrics (3) and (13) to describe a Klein bottle hole we must embed it in a three-dimensional Euclidean space with cylindrical coordinates at fixed time $t[6]$ whose metric can be written as

$$
d s^{2}=d z^{2}+d r^{2}+r^{2} d \phi^{2}=\left[1+\left(\frac{d z}{d r}\right)^{2}\right] d r^{2}+r^{2} d \phi^{2},
$$

However, since $r_{i}$ and $\varphi_{1}$ are no longer independent to each other, one can always convert metric (3) into a line element which is embeddible in the cylindrical space (17). That conversion can be made by first obtaining the expression for the variation of $r_{i}$ with respect to $\varphi_{1}$, that is

$$
\frac{d r_{i}}{d \varphi_{1}}=Q(i)=-\frac{\left[m_{i}\left(A_{i}-C_{i}\right)+n_{i}\left(B_{i}-D_{i}\right)\right] \sin \left(i \varphi_{1} / 2\right)}{2 i r_{i}}
$$

From which we get

$$
\begin{aligned}
& d s^{2}=-e^{2 \Phi} d t^{2}+\theta\left(2 \pi-\phi_{1}\right)\left(\frac{c(1) d r_{1}^{2}}{1-b_{01}^{2} / b_{1}^{2}}\right. \\
& \left.+d(1) Q(1) d r_{1} d \varphi_{1}+d \Omega_{1}^{2}\right)+\theta\left(\varphi_{1}-2 \pi\right) \\
& \times\left(\frac{c(2) d r_{2}^{2}}{1-b_{02}^{2}}+d(2) Q(2) d r_{2} d \varphi_{1}+d \Omega_{2}^{2}\right),
\end{aligned}
$$

with $c(i)+d(i)=1$.

Taking now $d z=\left(d z / d r_{1}\right) d r_{1}+\left(d z / d \varphi_{1}\right) d \varphi_{1}$, for $\varphi_{1} \leq$ $2 \pi$ and $d z=\left(d z / d r_{2}\right) d r_{2}+\left(d z / d \varphi_{1}\right) d \varphi_{1}$, for $\varphi_{1}>2 \pi$ we can derive for any allowed value of $\varphi_{2}$,

$$
c(i)=1+2\left(1-\frac{b_{0 i}^{2}}{b_{i}^{2}}\right)-2 \sqrt{1-\frac{b_{0 i}^{2}}{b_{i}^{2}}} .
$$

Hence it follows that the metric for the non orientable Klein bottle hole which is embeddible in flat space is described by Eq. (19) with $c(i)$ as given by (20) and $d=1-c(i)$. Using these coefficients, metric (17) will be the same as metric (19) for constant values of $\varphi_{2}$ if we identify the coordinates $r, \phi$ of the embedding space with either coordinates $r_{1}, \phi_{1}$, for $\varphi_{1} \leq 2 \pi$, or the coordinates $r_{2}, \varphi_{1}$, for $\varphi_{1}>2 \pi$, and if we require the function $z$ to satisfy

$$
d z / d r_{i}=1+\left(1-\frac{b_{0 i}^{2}}{b_{i}^{2}}\right)^{-1}-\left(1-\frac{b_{0 i}^{2}}{b_{i}^{2}}\right)^{-1 / 2}
$$

for any value of $\varphi_{1}$,

$$
\frac{d z}{d \varphi_{1}}=\frac{1}{2} \sqrt{\left[R\left(\varphi_{2}\right)_{1}-r_{1}\right]\left[r_{1}-\rho\left(\phi_{2}\right)_{1}\right]}
$$

for $\varphi_{1} \leq 2 \pi$, and

$$
\frac{d z}{d \varphi_{1}}=\sqrt{\left[R\left(\varphi_{2}\right)_{2}-r_{2}\right]\left[r_{2}-\rho\left(\phi_{2}\right)_{2}\right]}
$$

for $\varphi_{1}>2 \pi$, where

$$
R\left(\varphi_{2}\right)_{1}=A_{1}-B_{1} \cos \varphi_{2}, \quad \rho\left(\varphi_{2}\right)_{1}=C_{1}-D_{1} \cos \varphi_{2}
$$

and

$$
R\left(\varphi_{2}\right)_{2}=C_{2}-D_{2} \cos \varphi_{2}, \quad \rho\left(\varphi_{2}\right)_{2}=A_{2}-B_{2} \cos \varphi_{2} .
$$

From these expressions and the requirement that non orientable Klein bottle holes be connectible to asymptotically flat space-time, one can deduce how the embeddible surfaces would flare at or around the hole throat. Thus, from Eq. (21), one obtains

$$
\begin{aligned}
& \frac{d^{2} r}{d z^{2}}=\frac{b_{0 i}^{2} r_{i}}{b_{i}^{3} n_{i}}\left(\frac{1}{\sqrt{1-b_{0 i}^{2} / b_{i}^{2}}}-1\right) \\
& \times\left(1+\frac{1}{1-b_{0 i}^{2} / b_{i}^{2}}-\frac{2}{\sqrt{1-b_{0 i}^{2} / b_{i}^{2}}}\right)^{-7 / 2},
\end{aligned}
$$

which is positive for $2 \pi-\varphi_{2}^{c}>\varphi_{2}>\varphi_{2}^{c}=\arctan \left(b_{i} / a_{i}\right)$ and negative for $-\varphi_{2}^{c}<\varphi_{2}<\varphi_{2}^{c}$. Thus, exactly to as it happens in the case of toroidal ringholes ${ }^{4,13}$, the embedding surface flares outward for $d^{2} r / d r^{2}>0$ and flares inward $d^{2} r / d r^{2}<0$. It is for this reason that a Klein bottle hole would generally behave like a diverging lens for $2 \pi-\varphi_{2}^{c}>\varphi_{2}>\varphi_{2}^{c}$, and like a converging lens for $2 \varphi_{2}^{c}<\varphi_{2}<\varphi_{2}^{c}$, even though, unlike in the ringhole case, these behaviors will also depend on the value of the angle $\varphi_{1}$ due to the non orientable character of the Klein bottle hole space-time, such as we shall show in some detail in what follows.

In order to investigate how the embedding surface flares at or around the throat as the angle $\varphi_{1}$ is varied, so making the manifold non orientable, we distinguish two cases. The first case corresponds to condition (22), from which we can derive 


$$
\frac{d^{2} \varphi_{1}}{d z^{2}}=\frac{\left[-2 r_{1}+R\left(\varphi_{2}\right)_{1}+\rho\left(\varphi_{2}\right)_{1}\right]\left[R\left(\varphi_{2}\right)_{1}-\rho\left(\varphi_{2}\right)_{1}\right] \sin \left(\varphi_{1} / 2\right)}{2\left\{\left[R\left(\varphi_{2}\right)_{1}-r_{1}\right]\left[r_{1}-\rho\left(\varphi_{2}\right)_{1}\right]\right\}^{2}}
$$

Now, since $a_{i}>b_{i}$ for $0 \leq \varphi_{1} \leq 2 \pi$ one obtains that this expression vanishes at $\varphi_{1}=\pi$ and becomes negative for $\varphi_{1}<\pi$, on which values the embedding surface flares toward larger values of the radius $b_{1}$, and negative for $\varphi_{1}>\pi$ on whose values the embedding surface flares toward smaller values of $b_{1}$.

The second case corresponds to condition (23) for which we get

$$
\frac{d^{2} \varphi_{1}}{d z^{2}}=\frac{\left[-2 r_{2}+R\left(\varphi_{2}\right)_{2}+\rho\left(\varphi_{2}\right)_{2}\right]\left[R\left(\varphi_{2}\right)_{2}-\rho\left(\varphi_{2}\right)_{2}\right] \sin \left(\varphi_{1}\right)}{4\left\{\left[R\left(\varphi_{2}\right)_{2}-r_{2}\right]\left[r_{2}-\rho\left(\varphi_{2}\right)_{2}\right]\right\}^{2}}
$$

The critical value of $\varphi_{1}$ becomes then $\varphi_{1}=5 \pi / 2$. For $\varphi_{1}<5 \pi / 2$, Eq. (28) becomes negative so that the embedding surface flares toward smaller values of $b_{2}$, while it becomes positive for $\varphi_{1}>5 \pi / 2$, for which the embedding surface flares toward larger values of $b_{2}$.

The above analysis leads us to expect lensing effects to occur on the mouths of the nonorientable Klein bottlehole with respect to a bundle of light rays, at or near the throat, coming from the distribution of negative and positive values for the energy density [6]; that is, the mouths would act like a diverging lens for world lines along the values of the coordinates, at or near the throat, which correspond to negative energy density, and like a converging lens for world lines passing through regions with positive energy density. In order to confirm with full accuracy which regions around the throat behave like a converging or diverging lens, one must consider the nullray propagation governed by the integral of the stressenergy tensor. From the Einstein equations [6] it can be obtained that the mouths focus or defocus a bundle of rays, depending on the sign of the integral [6]

$$
\begin{aligned}
& I=\int_{\ell_{i}^{1}}^{\infty} d \ell_{i} e^{\Phi}\left(\rho c^{2}-\sigma\right)=\int_{\ell_{i}^{1}}^{\infty} d \ell_{i} e^{\Phi}\left(\frac{n_{1}}{r_{1}}\right)^{3}\left(T_{0}^{0}-T_{1}^{1}\right)=\int_{\ell_{i}^{1}}^{\infty} d \ell_{i} e^{\Phi} \frac{c^{4} b_{01}^{2} n_{1}^{2}}{16 \pi G b_{1}^{3} r_{1}^{2}} \times \\
& \left\{\frac{\frac{8 m_{1}}{a_{1}}-\left(\frac{A_{1}-C_{1}}{a_{1}}+\frac{B_{1}-D_{1}}{b_{1}}\right) \cos ^{2}\left(\frac{\varphi_{1}}{4}\right)+\frac{m_{1}^{(0)}}{m_{1}}+\frac{n_{1}^{(0)}}{n_{1}}-2}{4\left\{m_{1}^{2}+\frac{1}{4}\left[M_{1}\left(A_{1}-C_{1}\right)+N_{1}\left(B_{1}-D_{1}\right)\right] \cos ^{2}\left(\frac{\varphi_{1}}{4}\right)\right\}}+\frac{2\left(1+\sin \varphi_{2}\right)}{n_{1} b_{1}}-\frac{4 \cos \left(\frac{\varphi_{1}}{2}\right) \sin \varphi_{2}}{\left(A_{1}-C_{1}\right) m_{1} \sin ^{2}\left(\frac{\varphi_{1}}{2}\right)}\right\}
\end{aligned}
$$

In what follows we shall restrict ourselves to consider the case $\Phi=0$ and the still realistic situation where $\varphi_{2}=\pi / 2$ and $a_{1}>>b_{1}$. We obtain then

$$
\begin{aligned}
& I=\frac{c^{4}}{16 \pi G}\left[\xi_{1}\left(\varphi_{1}\right)\left(\frac{\pi}{2}-\arctan \left(\frac{\ell_{1}^{1}}{b_{01}}\right)\right)\right. \\
& \left.+\xi_{2}\left(\varphi_{1}\right)\left(1-\frac{\ell_{1}^{1}}{\sqrt{\left(\ell_{1}^{1}\right)^{2}+b_{01}^{2}}}\right)\right]
\end{aligned}
$$

where

$$
\xi_{1}\left(\varphi_{1}\right)=\frac{\left(B_{1}-D_{1}\right) \cos ^{2}\left(\frac{\varphi_{1}}{4}\right)+B_{1}}{a_{1}^{2}\left[4 a_{1}^{2}+\left(A_{1}-a_{1}\right)\left(a_{1}-C_{1}\right)\right]}
$$

$\Gamma$

$$
\xi_{2}\left(\varphi_{1}\right)=\frac{4}{a_{1}^{2}}
$$

We can conclude in this way that the integral $I$ is always definite positive, irrespective of the value of the angle $\varphi_{1}$. It follows that the dependence of $I$ on $\varphi_{1}$ will only contribute the strength of the action of the two different gravitational lenses that can be distinguished at and around the Klein-bottle hole throat, so just quantitatively modifying the behavior governed by the angle $\varphi_{2}$.

Inspection on the above equations leads to the final result which is twofold. On the one hand, we can derive that the surface gravity $\kappa$ is definite positive for $2 \pi-\varphi_{2}^{c}>$ $\varphi_{2}>\varphi_{2}^{c}$ and definite negative for $-\varphi_{2}^{c}<\varphi_{2}<\varphi_{2}^{c}$, and 


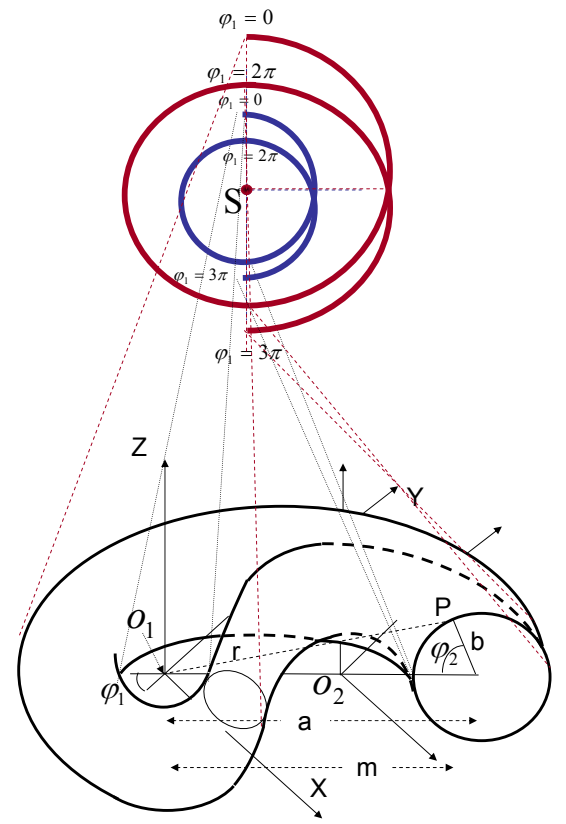

FIG. 3: Gravitational lensing effect produced by a Klein bottle hole from a single luminous source. On the lower part of the figure the parameters defining the Klein bottle hole throat in terms of which metric (3) is defined. The rays passing near the outer and inner surfaces respectively flare outward and inward, leading to a image from a luminous point placed behind the Klein bottle hole which is made of two concentric truncated bright spirals.

hence, since generally we have $T=-\left.\kappa\right|_{b_{i}=b_{0 i}} / 2 \pi$, that the Klein bottle hole emits thermal (phantom) radiation at negative temperature from the first of these regions and thermal radiation at positive temperature from the second region [16]. On the other hand, a ready calculation leads us to obtain (see Fig. 3) that the signature that a Klein bottle hole would in any event leave from a luminous source placed beyond it for an observer on earth is that of two concentric truncated double spiral.

In this case, besides valuable information on dark matter, dark energy and universe curvature or the very existence of Klein bottle holes in our universe, what could eventually be most astonishing in its implications would be an unprecedented insight into some of the contents of other universes linked to ours by means of these Klein bottle holes. After all, a Klein bottle hole is a perfectly valid solution to the Einstein equations for stress-energy tensor containing a given proportion of exotic stuff - possibly phantom energy- which is becoming more and more familiar in the full context of current cosmology [17]. The potentially attainable insight from such an interpretation is twofold. On the one hand, we would get a direct evidence for the existence of Klein bottle holes and, by the way, possibly of wormholes and ringholes, and on the other hand, we could have found a way to open the door to observe a parallel universe, and hence got a first direct evidence for the existence of the multiverse scenario.

There is an observation which may in principle distinguish a static Klein bottle hole staying within our own universe and having its two mouths at rest with respect to each another, from a Klein bottle hole that connect our universe to a parallel universe or, in general, to other universe of a multiverse scenario. In the latter case since there is no common space-time for the two universes (parallel or not), the two mouths should necessarily be in perpetual quasi periodic relative random motion with completely unspecified speed. This would make the time and space for the two universes at all independent because the relative motion of the two mouths converts the Klein bottle hole in a time machine that contains completely arbitrary closed timelike curves. In the case of the inner static Klein bottle hole, if the luminous source is kept motionless and the Klein bottle hole does not behave like a time machine, the double truncated spirals would be well resolved and defined on the pattern. However, if the positions of the two mouths continuously vary relative to each other in a random though quasi periodic way then the width of each of the truncated spirals would be stretched out and their resolution spoiled and clearly blurred due to the continuous and completely arbitrary changes of distance between the two mouths, thus leading to a glowing background around the spirals of Fig. 3, showing just a maximum of intensity at the average relative position of the mouths, provided the relative motion keep a sufficiently high degree of periodicity. In the latter case, the metric of the Klein bottle hole would change to be given by a line element that describes arbitrary time travel induced by a nearly periodic relative motion between the two mouths. Using arguments similar to those employed in Refs. [4] and [5] we finally get for that line element

$$
\begin{aligned}
& d s^{2}=-e^{2 \Phi} d t^{2}+\theta\left(2 \pi-\varphi_{1}\right)\left(\left\{-\left[1+\bar{g} F\left(\ell_{1}\right) \ell_{1} \sin \varphi_{2}\right]^{2}\right\}\right. \\
& \left.\times e^{2 \Phi} d t^{2}+c(1) d \ell_{1}^{2}+d(1) Q(1) d r_{1} d \varphi_{1}+d \Omega_{1}^{2}\right) \\
& +\theta\left(\varphi_{1}-\pi\right)\left(\left\{-\left[1+\bar{g} F\left(\ell_{2}\right) \ell_{2} \sin \varphi_{2}\right]^{2}+1\right\}\right. \\
& \left.\times e^{2 \Phi} d t^{2}+d(2) Q(2) d r_{2} d \varphi_{1}+d \Omega_{2}^{2}\right)
\end{aligned}
$$

where $\bar{g}=\bar{\gamma}^{2} \frac{d \bar{v}}{d t}$ is the average acceleration of the moving mouth, with $\bar{v}$ the corresponding average velocity, and $\bar{\gamma}=1 / \sqrt{1-\bar{v}^{2}}$ is the average on the fuzzy relativistic factor; finally $F(\ell)$ is a form factor that vanishes in the half of the Klein bottle hole which is assumed to be kept motionless, and rises up on average from 0 to 1 as one moves along the direction of the moving mouth. We must finally point out that any ringhole which is a time machine even within our universe will also show an defocused double truncated spiral pattern though not so blurred perhaps as that corresponding to an inter-universe ringhole. 


\section{FURTHER COMMENTS}

We finally briefly discuss the odds of finding a macroscopic Klein bottle hole which is kept stable. It was first argued [18] that only quantum wormholes, and hence quantum ringholes and Klein bottle holes, with nearly the Planck size can be stable, with larger tunnelings being violently destabilized by quantum effects produced by catastrophic particle creation taking place near the chronology horizons. Actually, Hawking even advanced his chronology protection conjecture [19] for wormholes which can also be applied to ringholes and Klein bottle holes, preventing the appearance of closed timelike curves, so making the universe safe for historians and free of the occurrence of the kind of phenomena dealt with in this paper. Thus, neither wormholes or ringholes nor Klein bottle holes could exist due to these quantum fluctuation instabilities.

However, besides some counter-examples to the Hawking's conjecture that includes e.g. some compelling argument by $\mathrm{Li}$ and Gott [20], it has been shown [21] that macroscopic wormholes, ringholes and Klein bottle holes can be stabilized after the cosmic coincidence time taking place at the onset of the dark energy era by the accelerating expansion of the universe which induces their throat to quickly growing comovingly to the super-luminal universal expansion. On the other hand, similarly to as it happens with wormholes [22], accretion of phantom energy onto the ringholes and Klein bottle holes should also induce in them a ultra rapid swelling up that would circumvent the kind of quantum effects considered by Hawking so that, such as it also happens with their above-mentioned size increasing which is comoving to the universal expansion, the destabilizing quantum ef- fects here cannot act in time to destroy the tunnel during the current speeding-up of the universe. Therefore, the odds for all of these tunnelings to exist and gravitationally act on the light coming from luminous sources the way we showed before appear to be good enough in the context of our accelerating universe as for allowing the kind of interpretation considered in this letter. On the other hand, in spite of the feature that for ringholes this interpretation just requires two objects aligned on axis, as opposed to requiring three objects to be on axis as the interpretation first suggested in Ref. [14] did, one could likewise think that possibly ringholes are quite rarer than galaxies so that the former interpretation is far from being quite more likely than the latter interpretation either. Thus, at least for the time being, it appears hard to decide which of these two interpretations should be chosen. Only more accurate analysis on the involved spectra and on the relative brightness of the two rings, and mainly the discovery of other double rings systems, could be used to finally choose which among these two interpretations is more likely to hold. As to using Klein bottle holes to check the physical existence of other universes, it appears just a matter of time to find a double truncated spiral blurred enough to clearly show a connection with other universes.

\section{Acknowledgments}

This work was supported by MICINN under research project no. FIS2008-06332. The author benefited from discussions with C. Sigüenza of the Estación Ecológica de Biocosmología of Medellín, Spain.
[1] M.S. Morris, K.S. Thorne, and U. Yurtsever, Phys. Rev. Lett. 61, 1446 - 1449 (1988); M.S. Morris and K.S. Thorne, Am. J. Phys. 56, 395 - 412 (1988); J.G. Cramer, R.L. Forward, M.S. Morris, M. Visser, G. Benford and G.A. Landis, Phys. Rev. D51 (1995) 3117.

[2] P.F. González-Díaz, Phys. Rev. D56, 6293 - 6297 (1997)

[3] A.A. Shatskiy, astro-ph/0712.2572 ; astro-ph/0809.0362; Astron. Rep. 51, 81 - 86 (2007) [Astron. Zh. 84, 99 - 104 (2007); A.A. Shatskiy, I.D. Novikov, and N.S. Kardashev, Phys.-USP 51, 457 - 464 (2008).

[4] P.F. González-Díaz, Lensing effects in ringholes and the multiverse in: Proceedings of the Workshop on "Cosmology, Quantum Vacuum, and Zeta Functions", ICE, CSIC, UAB, Barcelona (2010) (to be published); P.F. González-Díaz, Is the 2008 NASA/ESA double Einstein ring actually a ringhole signature?, astro-ph/1001.3778 .

[5] Whereas, as reported by M. Chown in New Scientist, 24 Sept. 2007, L. Mersini-Houghton and R. Holman attributed an observed big void in our universe to an entanglement effect caused by other universe within the context of string theory, A. Aguirre, M.C. Johnson and A.
Shomer considered the observable signatures left by other bubble universe which collided with our universe during their primordial evolution, in Phys. Rev. D76 (2007) 063509.

[6] P.F. González-Díaz and L.J. Garay, Phys. Rev. D59 (1999) 064026 .

[7] See contributions in Universe or Multiverse, edited by B. Carr (Cambridge Univ. Press, Cambridge, UK, 2007) .

[8] A.D. Linde and V. Vanchurin, How many universes are in the multiverse hep-th/0910.158 v2; A. Linde, Mod. Phys. Lett A1, 81 - 85 (1986); Phys. Lett. B175, 395 400 (1986) .

[9] H. Everett, Rev. Mod. Phys. 29, 454 - 462 (1957); See papers by H. Everett III, J. A.Wheeler and B. S. De- Witt in: The Many-Worlds Interpretation of Quantum Mechanics, edited by B. S. DeWitt and N. Graham. (Princeton Univ. Press, Princeton, USA, 1973); K. Ashok and M. Douglas, JHEP 060 065 (2004) 0401 arXiv:hep-th/0307049; L. Susskind, arXiv:hep-th/0302219; S. Robles-Pérez, P. MartíMoruno, A. Rozas-Fernández and P.F. González-Díaz, Class. Quant. Grav. 24, F41 - F44 (2007) . 
[10] See paper by Martin Rees in Ref. 7.

[11] R. R. Caldwell, Phys. Lett. B 545, 23 - 29 (2002); S. M. Carroll, M. Hoffman and M. Trodden, Phys. Rev. D 68 (2003) 023509; R. R. Caldwell, M. Kamionkowski and N. N. Weinberg, Phys. Rev. Lett. 91 (2003) 071301; P.F. González-Díaz, Phys. Lett. B 586, 1 - 4 (2004); P.F. González-Díaz, Phys. Rev. D 69 (2004) 063522 .

[12] A. Einstein, Science 84, 506 - 507 (1936).

[13] P.F. González-Díaz, Phys. Rev. D54, 6122 - 6131 (1996).

[14] R. Gavazzi, T. Treu, L.V. E. Koopmans, A.S. Bolton, L.A. Moustakas, S. Burles and P.J. Marshall, The Sloan Lens ACS Survey. VI: Discovery and analysis of a double Einstein ring, astro-ph/0801.1555 .

[15] M. Abramowitz and I.A. Stegun, Handbook of Mathematical Functions, (Dover, New York, USA, 1972).

[16] The ultimate physical reason why a Klein bottle hole may radiate phantom energy at a negative temperature lies on the feature that the surface embedding the Klein bottle hole space-time flares outward at or near the throat on the region $\varphi_{1}<\varphi_{1}^{h}$, see P. Martín-Moruno and P.F. González-Díaz, Phys. Rev. D80, 024007 (2009); Clas.
Quant. Grav. 26, 215010 (2009).

[17] D. J. Mortlock and R. L. Webster, The statistics of wide-separation lensed quasars, Mon. Not. Roy. Astron. Soc. 319, 872 - 878 (2000) arXiv:astro-ph/0008081; A. G. Riess et al. [Supernova Search Team Collaboration], Astron. J. $116 \quad$ (1998) 1009-1038 arXiv:astro-ph/9805201]; S. Perlmutter et al. [Supernova Cosmology Project Collaboration], Astrophys. J. 517, 565 - 586 (1999) arXiv:astro-ph/9812133; J. L. Tonry et al. [Supernova Search Team Collaboration], Astrophys. J. 594, 1 - 24 (2003); D. N. Spergel et al. [WMAP Collaboration], Astrophys. J. Suppl. 148, 175 - 194 (2003); C. L. Bennett et al., Astrophys. J. Suppl. 148, 1 - 27 (2003);

[18] P.F. González-Díaz, Phys. Rev. D58 (1998) 124011.

[19] S.W. Hawking, Phys. Rev. D46, 603 - 611 (1992).

[20] Li-Xin-Li and J. R. Gott, Phys. Rev. Lett. 80 (1998) 2980 - 2983 (1998).

[21] P.F. González-Díaz, Phys. Rev. D68 (2003) 084016.

[22] P.F. González-Díaz, Phys. Rev. Lett. 93 (2004) 071301. 